\title{
On coupling cellular automata based activation and finite element muscle model applied to heart ventricle modelling
}

\author{
R. Cimrman, J. Kroc, E. Rohan, J. Rosenberg \& Z. Tonar \\ New Technologies Research Centre, University of West Bohemia, 30614 \\ Plzeñ, Czech Republic
}

\begin{abstract}
The paper deals with the finite element macroscopic model of contracting myocardium. The constitutive model of the tissue is based on the theory of mixtures, involving a simple model of intramyocardial blood perfusion. Anisotropy of the tissue is described in terms of preferential directions of the muscle and connective fibres. A simple Hill-type model of the muscle fibre contraction is employed. The local value of the activation parameter is defined through the four phases of the action potential wave; its propagation is simulated using the cellular automaton (CA). The approximated excitable medium, which comprises the conducting system, is partitioned into the normal, conducting and void cells. The coupling of the CA analysis with the FEM model of the heart mechanics is discussed. Introductory numerical examples are included, describing contraction of the dog heart.
\end{abstract}

\section{Introduction}

The aim of this paper is to present a computational model of the excitable myocardial tissue. Although this topic has become a subject of intensive research in recent decade, cf. $[7,1,3]$, the existing models usually do not capture the complexity of the phenomena which feature modelling of the myocardium mechanics. For realistic simulations of heart beats it is important to treat not only the 3D geometrical structure of the cardiac muscle fibres, but also a relevant model of the excitable medium. In our approach the latter is based on the cellular automaton (CA) which is employed to describe spatial propagation of the depolarization wave. The $\mathrm{CA}$ is coupled with FE model of the deformable ventricles. 


\section{6}

Simulations in Biomedicine $V$

The CA - FEM coupling is tested in a simulation of deforming dog-heart ventricles. So far in our model we have considered only a static, spatially uniform pressure load of the ventricles and computed the CA response to define activation of the muscle fibres in time over the $3 \mathrm{D}$ geometry. For a more genuine description of the heart beats the fluid flow problem inside the ventricles should be considered as well as a correct interaction of the blood with the ventricular walls. This, however, is beyond purpose of this study which should show only viability of the approach presented.

\section{Structure and functions of the myocardium}

Cardiac muscle is the striated type of muscle tissue with contractile myofibrils regularly arranged in the form of sarcomeres, cf. [4]. The ventricular muscle is arranged into three systems of fibres. Whereas the inner and outer layers are thin muscle membranes, the middle layer consists of locally parallel muscle fibres that are organized into twisted sheets immersed in the collagenous matrix. The electrophysiological discontinuity between the atrial and ventricular myocardial masses is ensured by the fibrous skeleton, which also serves as a stable but deformable base for the attachments of the muscle fibres.

\subsection{Excitatory and conductive system of the heart}

The heart is endowed with a special system for generating rhythmical impulses and conducting these impulses rapidly throughout the heart. Its elements are derived from cardiac muscle cells containing few contractile fibrils and they exhibit selfexcitation with inherent rhythmicity and varying rates of conduction. The conduction velocities are shown in Table 1 .

The sinus node displays self-excitation acting as a normal pacemaker. Then the impulse is conducted to the atrioventricular (A-V) node, where the action potential (AP) wave from the atria is delayed before passing into the ventricles. This delay allows the atria to empty their contents into the ventricles before ventricular contraction begins. The A-V bundle, conducting the impulse from the atria into the ventricles, admits only forward conduction, preventing re-entry of cardiac impulses by this route from the ventricles to the atria. The left and right bundle branches and the system of large subendocardial Purkinje fibres conduct the impulse immediately to all portions of the ventricles, thus, preserving synchronous contraction required for the effective pressure generation.

Cardiac muscle is refractory to restimulation during the AP wave, when a normal cardiac impulse cannot re-excite an already excited muscle area. The absolute refractory period of the ventricle is $0.25-0.3 \mathrm{~s}$, which is about the duration of the AP. During an additional relative refractory period of about $0.05 \mathrm{~s}$, the muscle is excitable by a supranormal impulse only. 
Table 1: Conduction velocities of the action potential in the heart [4]

\begin{tabular}{|c|c|}
\hline Part of the conducting system & Velocity of conduction $\left[\mathrm{m} . \mathrm{s}^{-1}\right]$ \\
\hline atrial muscle and internodal pathways & $0.3-1.0$ \\
\hline$A-V$ node and penetrating $A-V$ bundle & $0.02-0.05$ \\
\hline left/right bundle branches and Purkinje fibres & $1.5-4.0$ \\
\hline ventricular muscle & $0.3-0.5$ \\
\hline
\end{tabular}

\subsection{Excitation-contraction coupling and function of calcium ions}

The action potential initiates release of $\mathrm{Ca}^{2+}$ ions from the sarcoplasmic reticulum and their diffusion from the $\mathrm{T}$ tubules, thus enhancing the contraction power. The $\mathrm{Ca}^{2+}$ ions diffuse into the system of myofibrils and catalyze the chemical reactions in the troponin-tropomyosin complex that promote sliding of the actin and myosin filaments along one another; this produces the muscle contraction.

At the end of the plateau of the AP, the influx of $\mathrm{Ca}^{2+}$ to the interior of the muscle fibre is suddenly cut off, and the $\mathrm{Ca}^{2+}$ ions are rapidly pumped back from the sarcoplasm. As a result, the contraction ceases until a new action potential occurs.

\section{Macroscopic model of the heart tissue}

\subsection{Application of the mixture theory approach}

A detailed description of the heart tissue mechanics from the sub-microscopic to macroscopic scales is beyond our capabilities; such a model would be too complex and, thus, expensive to be used for practical simulations. The model presented below reflects main anisotropic features of the tissue, which can be observed by a standard analysis of micrographs. In this way volume fractions can be determined for major heart tissue components which form the tissue fibrous skeleton and connective tissue matrix. These fibrous components are assigned by $\alpha$; they are responsible for anisotropy of the tissue. Therefore, it is important to determine also one or more preferential directions for each component $\alpha$ and the associated volume fractions. Let $N_{\mathrm{PFD}}$ be the total number of preferential directions for all fibrous components $\alpha=1,2, \cdots, N_{\mathrm{FC}}$. We define the index subsets $I_{\alpha}$, so that $\left\{1, \ldots, N_{\mathrm{PFD}}\right\}=\bigcup_{\alpha=1}^{N_{\mathrm{FC}}} I_{\alpha}$. By $\phi^{k}$ we denote a volume fraction associated with the preferential direction $\nu^{k}$. Denoting by $\phi_{m}$ the volume fraction of the amorphous matrix, we have

$$
\phi_{m}+\sum_{\alpha=1}^{N_{F C}} \sum_{k \in I_{\alpha}} \phi^{k}=1 .
$$

We assume that any interpenetration of the components is negligible, so that a unique field of displacements can be considered. It possesses a unique macro- 
scopic strain field; denoting by $E_{i j}$ the Green-Lagrange strain tensor, we define the projected strain for each $k=1, \ldots, N_{\mathrm{PFD}}$ :

$$
\epsilon^{k}=E_{i j} \nu_{i}^{k} \nu_{j}^{k}, \text { no summation over } k .
$$

Using $\epsilon^{k}$ we can define tension $\tau^{k}$ in the preferential direction of the $k$-th component. In general, the constitutive law depends on internal variables $\chi_{1}, \chi_{2}, \ldots$ and activation parameters $a_{1}, a_{2}, \ldots$ (in muscle fibres). Thus, for the $k$-th component the corresponding 2 nd Piola-Kirchhoff stress is given by

$$
T_{i j}^{k}=\nu_{i}^{k} \nu_{j}^{k} \tau^{k}, \quad \text { where } \tau^{k}=f^{k}\left(t, \epsilon^{k}, \dot{\epsilon}^{k},\{\chi\},\{a\}\right),
$$

where $t$ is time and $\dot{\epsilon}$ is the strain rate. If $k$ is associated with a collagen fibre, then $f^{k}$ is introduced as a viscoelastic response, involving one internal variable. In our model we employ the three parametric model of the standard solid element with a nonlinear elastic response.

If $k$ is associated with a muscle fibre, then one, or more activation parameters $a_{1}, a_{2}$ depend on the local value of the excitation wave. The muscle contraction force can be described using the sliding cross-bridge theory with approximation of the distribution moments, cf. [10]. In this case (3) involves three internal parameters $\chi_{0}, \chi_{1}, \chi_{2}$ which correspond to the 0 th, 1 st and 2 nd moments of the crossbridge distribution; these obey the differential equations

$$
\dot{\chi}_{l}=g_{l}\left(\epsilon, \dot{\epsilon}, a,\left\{\chi_{0}, \chi_{1}, \chi_{2}\right\}\right), l=0,1,2,
$$

where $\epsilon$ is the projected strain (2). Thus, $\{\chi\}$ are associated with the particular preferential direction.

In this study we use a model of the Hill type, which does not involve internal variables and, thus, cannot describe fading memory effects. The generated tension $\tau$ of (3) is defined as follows:

$$
\tau\left(\epsilon, \dot{\epsilon}, F_{\mathrm{a}}\right)=\eta \dot{\epsilon}+F_{\mathrm{a}} \bar{F}_{\max } \exp \left\{-\left(\frac{\epsilon-\varepsilon_{\mathrm{opt}}}{s}\right)^{2}\right\} \exp \{\kappa \min \{0 ; \dot{\epsilon}\}\},
$$

where $s$ determines the sensitivity of the actin-myosin overlaps with respect to difference of deformation $\epsilon$ and the optimal deformation $\varepsilon_{\text {opt }}, \kappa$ relates to behaviour while shortening and $\eta$ is a viscosity coefficient. For more information about this and similar models, see [8]. The value of the activation parameter $F_{\mathrm{a}} \in[0,1]$ should correspond to a propagation of an action potential in heart. This can be done using e.g. the Fitz-Hugh Nagumo equations, or using the theory of cellular automata, see Section 4.

The amorphous matrix of the tissue can be described as a hyperelastic material. The effective part of the stress $S_{i j}^{m}$ is defined using energy function $W^{m}\left(E_{i j}\right)$, i.e. $S_{i j}^{m}=\partial W^{m} / \partial E_{i j}$. A unique thermodynamic pressure field $p$ corresponds with (in)compressibility of the bulk tissue. In the next section we discuss the blood 
perfusion problem where $p$ acts as the intramyocardial pressure. By virtue of the mixture theory the total 2nd Piola-Kirchhoff stress in the tissue is

$$
S_{i j}=-J C_{i j}^{-1} p+\phi_{m} \frac{\partial W^{m}}{\partial E_{i j}}+\sum_{\alpha=1}^{N_{\mathrm{FC}}} \sum_{k \in I_{\alpha}} \phi^{k} T_{i j}^{k},
$$

where $C_{i j}=2 E_{i j}+\delta_{i j}$ is the right Cauchy-Green deformation tensor and $J=$ $\left(\operatorname{det} C_{i j}\right)^{1 / 2}$.

The model enables that at any material point both the passive and the active fibres can be defined in several preferential directions, as required in accordance with the histological observation. The fibres are defined to have no stiffness opposing their compression, which corresponds to the buckling phenomenon of fibres dispersed in the matrix at the microstructure. It amplifies anisotropy of the composite, making the difference between tension and compression. As another consequence it allows for describing creep and relaxation of unloaded viscoelastic fibres when the matrix is being compressed in the direction of the fibres.

\subsection{Intramyocardial blood perfusion}

The blood perfusion of the myocardial muscle presents the most important source of the energy which is needed for muscle fibres to contract and, thus, to pump the blood. The perfusion is maintained thanks to the coronary arteries which form a network branching in the epicardium and entering the deeper layers.

Neglecting the hierarchical structure of the intracoronary system, here we shall consider only a crude approximation of the perfusion which is based on the macroscopic Darcy's flow, see [1]. Using this approach, however, we should be able to account for the periodic changes of the bulk volume of the myocardial tissue, as dependent on shrinkage and distension processes during one cardiac cycle. The local compression of the myocardium can be a measure of the blood supply. In consequence, this can be used as a feedback which influences the local propagation of the activation wave.

The apparent perfusion velocity $w_{i}$ depends on the gradient of the intramyocardial pressure

$$
w_{i}=-K_{i j} \frac{\partial p}{\partial x_{j}}, K_{i j}=k \delta_{i j}\left(\frac{J-1}{N_{f}}+1\right)^{2},
$$

where $k$ is the permeability, and $N_{f}$ the porosity, [1]. The local conservation of mass is expressed as

$$
\frac{\partial \dot{u}_{i}}{\partial x_{i}}+\frac{\partial w_{i}}{\partial x_{i}}=0 \quad \text { in } \Omega(t)
$$

where $\dot{u}_{i}$ is the material derivative of the displacements. In order to write (8) in the weak form, the boundary conditions for $p$, or $\partial p / \partial x_{i}$ must be specified. On the epicardium the blood can be squeezed out to the coronary system (or sucked in), $p=p_{0}$ on $\partial \Omega_{\text {epicard }} ; p_{0}$ should correspond to the venous pressure. As no blood is assumed to seep into the ventricle, or through the basement, $\partial p / \partial x_{i}=0$ on 
$\partial \Omega \backslash \partial \Omega_{\text {epicard. }}$ After transformation to the reference domain $\Omega(0)$ and using some other manipulations we obtain the weak formulation

$$
\int_{\Omega(0)} \dot{j} q d X+\int_{\Omega(0)} \bar{K}_{i j} \frac{\partial p}{\partial X_{j}} \frac{\partial q}{\partial X_{i}} d X=0, \forall q \in Q(\Omega(0))
$$

where $Q(\Omega)=\left\{q \in H^{1}(\Omega) \mid q=0\right.$ on $\left.\partial \Omega_{\text {epicard }}\right\}, \dot{J}=J\left(C^{-1}\right)_{i j} \dot{E}_{i j}$ and the transformed permeability tensor is $\bar{K}_{i j}=k J\left(C^{-1}\right)_{i j}\left((J-1) / N_{f}+1\right)^{2}$. Here (9) replaces the usual incompressibility equation which is comprised in the mixed formulation for computing the displacement and pressure fields.

\subsection{FE discretization and the energy conserving scheme}

In this scheme [2] the quantities are evaluated at a "mid-point" $m$ between the time levels $t-1$ (known) and $t$ (unknown). We denote a quantity at times $t-1$, $t$ by $X_{1}, X_{2}$ respectively. Thus $X_{m}=w_{1} X_{1}+w_{2} X_{2}, w_{1}+w_{2}=1, w_{1} \epsilon$ $[0,0.5]$. By setting $w_{1}<0.5$ a numerical dissipation, which stabilises the solution, is introduced into the system. The finite element approximation of displacements and the pressure is given by $\underline{u} \approx \chi^{T} \cdot u, p \approx \psi^{T} \cdot p$ respectively, where $\chi$ and $\psi$ are the Galerkin basis functions.

In this paragraph we employ the following notation: $\boldsymbol{F}$ the deformation gradient, $J=\operatorname{det} \boldsymbol{F}, \boldsymbol{s}$ the $2^{\text {nd }}$ Piola-Kirchhoff stress tensor in vector form, $\boldsymbol{G}_{c}$ the pressure gradient operator, $C$ the right Cauchy-Green tensor in matrix form, $M$ the mass matrix, $\delta_{u} \boldsymbol{E}_{m}\left(\boldsymbol{u}_{m} ; \boldsymbol{v}_{m}\right)=\boldsymbol{B}_{m} \boldsymbol{v}_{m}$ the gradient of the Green strain. Using this notation, we can write the discrete equilibrium equation in the mid-point $m$ (omitting any loading terms for brevity):

$$
\begin{array}{r}
\boldsymbol{g}_{m}:=\int_{\Omega_{0}} \boldsymbol{B}_{m}^{T} \boldsymbol{s}_{m} \mathrm{~d} \Omega+\boldsymbol{M}\left(\frac{\dot{\boldsymbol{u}}_{2}-\dot{\boldsymbol{u}}_{1}}{\Delta t}\right)=0, \\
\boldsymbol{h}_{m}:=\int_{\Omega_{0}}\left(J_{1}-J_{2}\right) \boldsymbol{\psi} \mathrm{d} \Omega-\Delta t\left[\int_{\Omega_{0}} \boldsymbol{G}_{c}^{T} \overrightarrow{\boldsymbol{K}}_{1} \boldsymbol{G}_{c} \mathrm{~d} \Omega\right] \boldsymbol{p}_{2}=0 .
\end{array}
$$

Above we have replaced $\dot{J}$ of (9) by $\left(J_{2}-J_{1}\right) / \Delta t$ and related $\bar{K}_{i j}$ to step $t-1$ to simplify the tangent matrix. The tangent linear system of the Newton method then attains this form (using $\dot{u}_{2} \approx 2\left(u_{2}-u_{1}\right) / \Delta t-\dot{u}_{1}$ ):

$$
\left(\begin{array}{ccc}
\boldsymbol{K}_{t, m}+\frac{2}{\Delta t^{2}} \boldsymbol{M} & , & \boldsymbol{R}_{m} \\
\boldsymbol{P}_{m} & , & -\Delta t \boldsymbol{D}
\end{array}\right)\left(\begin{array}{c}
\delta \boldsymbol{u} \\
\delta \boldsymbol{p}
\end{array}\right)=\left(\begin{array}{c}
-\boldsymbol{g}_{m, \mathrm{old}} \\
-\boldsymbol{h}_{m, \text { old }}
\end{array}\right)
$$

where $\boldsymbol{K}_{t, m}$ is the usual tangent stiffness matrix, $\boldsymbol{R}_{m}=\partial \boldsymbol{g}_{m} / \partial \boldsymbol{p}_{2}, \boldsymbol{P}_{m}=\partial \boldsymbol{h}_{m} / \partial \boldsymbol{u}_{2}$ and $\boldsymbol{D}$ is the bracketed term of (11) $\left(=\partial \boldsymbol{h}_{m} / \partial \boldsymbol{p}_{2}\right)$. We set $\boldsymbol{u}_{2, \text { new }}=\boldsymbol{u}_{2, \text { old }}+$ $\delta \boldsymbol{u}, \boldsymbol{p}_{2, \text { new }}=\boldsymbol{p}_{2, \mathrm{old}}+\delta \boldsymbol{p}$ and iterate until convergence is achieved. 


\section{Modelling propagation of the excitation wave}

\subsection{Simulation by the cellular automata (CA)}

In the mathematical model of muscle fibres, the tension generated by these fibres depends on one, or more, activation parameters (functions of space and time), which have to be specified as an input. For simulations of complex problems, such as the myocardium contraction, these parameters must vary, as the "activation" propagates, reflecting the chemical and other processes in the tissue. A suitable modelling tool fitted for this purpose seems to be provided by the theory of cellular automata (CA), cf. $[6,9]$.

The concept of CA was developed already by John von Neumann in 1948. In fact, CA are discrete dynamic systems useful for simulations of complex problems, where a classical formulation in terms of (partial) differential equations is too difficult or does not exist.

CA-model discretizes space into three-dimensional (3D) lattice of cubes. Cubes, i.e. elements of this lattice, are called cells. Every cell has defined a neighbourhood - usually a list of nearest neighbouring cells and the cell itself - that is uniform through the whole lattice. Every cell contains a list of variables, e.g. state, morphology, etc. Morphology is divided into three different classes: conducting system, muscle, and the SA-node. The evolution of the system is driven by a transition rule that computes new values of variables using values of cells laying in the neighbourhood of given cell from the previous CA-step.

Generally, the transition rule can be split into several sequential steps that handle the evolution of different parts of the cellular automaton belonging into different morphological classes, i.e. the conducting system, muscle, and the SA-node. Due to computational reasons, one additional morphological class is defined with the empty value that should be understood in the following sense. A cell having the empty morphological state is not included into propagation of excitation events, but is important to define the neighbourhood; every surface cell has at least one cell with empty morphological state in its neighbourhood.

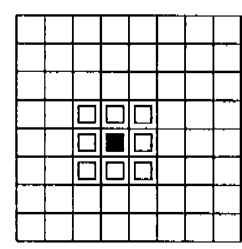

Figure 1: The next state in the current cell (filled rectangle) depends on its actual state and on states of the cells in the local neighbourhood.

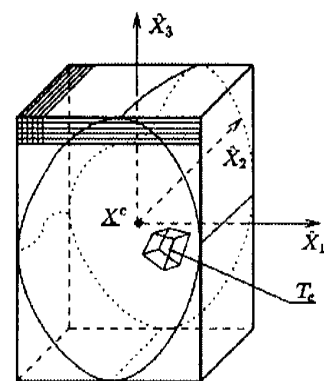

Figure 2: Rotated body with a selected element $T_{e}$, CA lattice. 
Each cell in the CA-model has two variables, namely, state and morphology. Variable state of the cell defines the level of excitation of the cell laying in the interval of $\langle 0,21\rangle$. State equal to the value of 21 is the excited state. As the value of the state variable decreases, first absolutely refractory states and then relatively refractory states are reached. The resting state, i.e. the situation when the cell waits for an excitation event, has assigned the zero value. Every cell inside the lattice belongs to one of the following morphology classes, i.e. empty, the conducting system, muscle, and the SA-node with values of $0,1,2$, and 3 , respectively. All cells with values between 1 and 3 define excitable medium. Cells with value equal to zero, i.e. empty cells, do not influence the excitation process at all.

\subsection{CA and FEM coupling}

The results of $\mathrm{CA}$ simulation serve for defining the activation parameters of the above mentioned model of muscle fibres.

Thus, modelling of muscle contraction is performed in two steps:

1. CA simulation for all required time steps.

2. FE simulation of muscle mechanics using the distribution of action potentials, as computed by CA.

As the result of step 1, at any time and for each integration point of the FE mesh the activation parameter $F_{a}$, see (5), is obtained by an averaging procedure which operates on the state values of all CA cells within the particular element.

This loose coupling is possible due to the assumed independence of the state of CA cells on deformation of the tissue at the corresponding point, as computed by FE simulation.

Clearly, the CA lattice must reflect the geometry of (undeformed) FE mesh. For this purpose the following algorithm has been developed:

- Input the box which is going to contain the CA lattice.To minimize the number of CA cells it is possible to rotate the coordinate system $\hat{X}_{i}$ of CA box with respect to the system $X_{i}$ of the FE body.

- Set division of CA box, i.e. cell counts in axial directions $\hat{X}_{i}$.

- For every element of FE mesh mark (as tissue, conducting system, pacemaker, ...) the CA cells $\underline{c}$ contained within, see Fig. 2. This step involves computing the reference element coordinates $\underline{\xi}=\underline{\xi}(\underline{c})$ by the Newton method (with $3 \times 3$ tangent matrix of the mapping $\underline{c}(\underline{\xi}) \approx \chi^{T}(\underline{\xi}) \cdot x$, where $x$ are coordinates of the element nodes).

\section{Numerical simulation of the ventricular contraction}

The numerical simulations were performed using the geometry of the dog heart, for which we obtained relevant geometrical data with a correct orientation of muscle fibres, see Fig. 3. We consider a model of the heart ventricles only (i.e. beneath the basal skeleton), which can be assumed to form a separate electro-mechanical subsystem of the heart. The ventricles (fixed at the top) are inflated by ventricular pressures reaching $120 \mathrm{~mm} \mathrm{Hg}$ for the left and $25 \mathrm{~mm} \mathrm{Hg}$ for the right ventricle, 


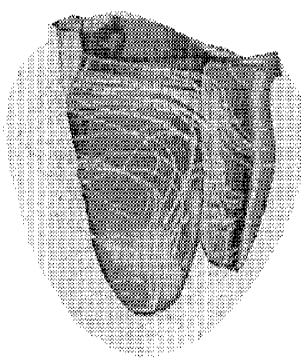

Figure 3: Geometry of the dog heart with outlined network of muscle fibres.

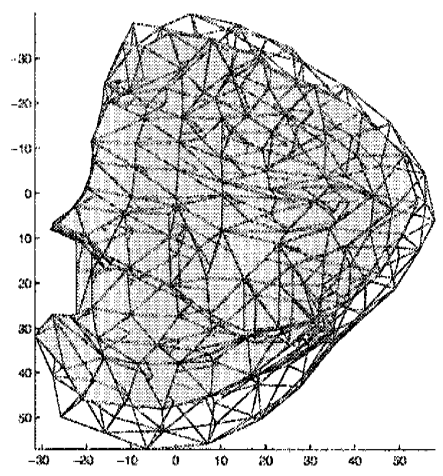

Figure 5: Contracted and inflated shapes of ventricles.

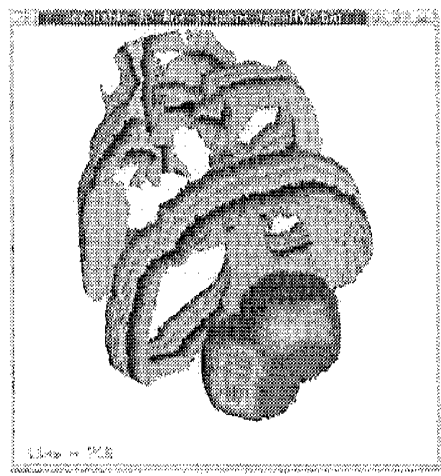

Figure 4: Propagation of the action potential. Three consecutive instants. 4 states indicated by different colours.
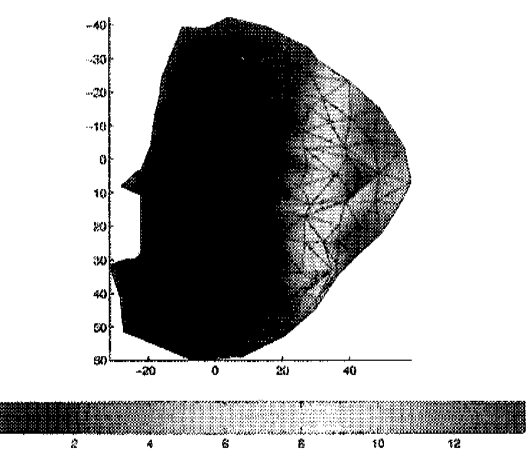

Tigure 6: Stress distribution in active (muscle) fibres.

respectively. Difference between contracted and released heart geometry is demonstrated in Fig. 5. In Fig. 6 the tension in muscle fibres is displayed for an initial period of the systolic contraction. The values of activation are obtained from the CA analysis of excitation. For illustration, in Fig. 4 we display results of CA analysis on the human heart, for which we have the conducting system well defined.

\section{Conclusion}

The model of myocardium presented in this paper allows for capturing important nonlinear and anisotropic features of the cardiac tissue. In order to describe behaviour of the pumping heart, a relevant model of the excitation wave propagation is needed. Our approach is based on coupling the cellular automata (CA), for simulation of the excitation, with the finite element model of the heart mechanics. The CA model seems to be a flexible tool, which can be adapted easily to approx. 
imate the wave propagation, influenced by various physiological effects. The simple model of muscle fibres currently used seems to be sufficient for simulation of steady state periodic responses. For studies regarding transition phenomena, caused e.g. arrhythmia, however, more sophisticated models based on cross-bridge kinetics can be used, which also involve fading memory effects. Due to inclusion of the myocardial intracoronary blood perfusion, its consequence on stiffening of the ventricular wall can be analyzed. Local deficiency in intramyocardial blood supply can decrease rapidly the speed of excitation wave; this effects can be captured by the $\mathrm{CA}$ algorithm.

For further improvement of the heart model the dynamic blood flow inside the ventricles will have to be pursued. Effects of the local tissue deformation on propagation of the excitation wave should also be reconsidered in forthcoming studies; for this, however, the CA simulation will need to be tightly coupled with the FE analysis of deformation.

Acknowledgment The research was supported by the project LNO0B084 of The New Technologies Research Centre, University of West Bohemia, Plzeň. We would also like to thank Frank Genot of INRIA Rocquencourt (France) for providing us the refined FE meshes of the dog heart based upon the original Auckland data.

\section{References}

[1] Campen, D.H., Huyghe, J.M., Bovendeerd, P.H.M. \& Arts, T., Biomechanics of the heart muscle. Eur. J. Mech., A/Solids, 13, pp. 19-41, 1994.

[2] Cimrman, R. \& Rohan, E., FE simulation of muscle behaviour in dynamic problems. Proc. of the conference ECCM 2001, Krakow, full paper on CDROM, 2001.

[3] Guccione, J.M., Costa, K.D. \& McCulloch, A.D., Finite element stress analysis of left ventricular mechanics in the beating dog heart. Journal of Biomechanics, 28, pp. 1167-1177, 1995.

[4] Guyton, A.C. \& Hall, J.E., Textbook of Medical Physiology. W.B. Saunders Company, Philadelphia, 1995.

[5] Holzapfel, G.A., Nonlinear Solid Mechanics. J. Wiley, Chichester, 2000.

[6] Chopard, B. \& Droz, M., Cellular Automata Modeling of Physical Systems. Cambridge University Press, Cambridge, 1998.

[7] Nash, M., Mechanics and material properties of the heart using an anatomically accurate mathematical model. Doctoral thesis, The University of Auckland, 1998.

[8] Rohan, E. \& Cimrman, R., Sensitivity analysis and material identification for activated smooth muscle. Comput. Assisted Meth. Engrg. Sci., 9, pp. 519-541, 2002.

[9] Vichniac, G.Y., Simulation physics with cellular automata, Physica 10D, pp. 96-116, 1984.

[10] Zahalak, G. I., A distribution-moment approximation for kinetic theories of muscular contraction. Mathematical Biosciences, 55, pp. 89-114, 1981. 